\title{
HAMILTONIAN FORMALISM FOR SPACE CHARGE DOMINATED BEAMS IN A UNIFORM FOCUSING CHANNEL*
}

\author{
A.Riabko, M.Ellison, X.Kang, S.Y.Lee, J.Y.Liu, D.Li, A.Pei, L.Wang \\ Department of Physics, Indiana University, Bloomington, IN 47405
}

\begin{abstract}
Halo formation for a test particle in a mismatched KV beam is studied. Parametric resonances of the particle Hamiltonian due to envelope modulation are studied with particular emphasis on period 2 resonance which plays dominant role in Halo formation. It is shown that the onset of global chaos exhibits a sharp transition when the amplitude of modulation is larger than a critical value which is a function of a single parameter, $\kappa$, i.e., the ratio of the space charge perveance to the focusing strength.
\end{abstract}

\section{INTRODUCTION}

The interest in intense charged particle beams has grown in past few years due to demand in high brightness and high intensity ion sources. For beams dominated by the self-space-charge force Kapchinskij and Vladimirskij have constructed a self consistent equilibrium distribution function which obeys the KV equation governed by the external focusing field and the self space charge force [1]. In order to understand the motion of a test particle in KV beam simultaneous numerical integration of envelope and particle equations of motion can be used. The assumption is that the envelope evolution will affect the particle dynamics, while the particle motion does not affect the envelope. The results of numerical simulations indicate that the halo formation arises mainly from resonance excitations [2-5].

This paper is organized as follows. In Sec. II, we review properties of the envelope Hamiltonian and study a motion of a weakly mismatched beam in a uniform focusing channel. In Sec. III, the particle Hamiltonian is separated into autonomous Hamiltonian and time dependent perturbation; the condition on existence of primary parametric resonances is obtained. Conditions for global chaos and estimation of halo diameter as function of modulation amplitude are considered. Conclusion is given in Sec. IV.

\section{THE HAMILTONIAN FOR THE ENVELOPE PHASE SPACE}

The KV envelope Hamiltonian for a uniform focusing channel is given by

$$
H_{e}=\frac{P^{2}}{2}+\frac{\mu^{2} R^{2}}{2}-K \ln (R)+\frac{\epsilon^{2}}{2 R^{2}}
$$

(where $\mu$ is the external focusing field, $\epsilon$ is the emittance of the beam and $K=\frac{2 N r_{c l}}{\beta^{2} \gamma^{3}}$ is the space charge perveance parameter).

\footnotetext{
*Work supported by grants from NSF PHY-9221402 and DOE DE-FG0293ER40801.
}

Using scaling transformation, we obtain the following expression for the envelope Hamiltonian

$$
H_{e}=\frac{P^{2}}{2}+\frac{R^{2}}{2}-2 \kappa \ln (R)+\frac{1}{2 R^{2}}
$$

where $\kappa=\frac{K}{2 \mu \epsilon}$. The matched beam radius $R_{0}$ and the frequency of small amplitude envelope oscillations $\nu_{e}$ are given by

$$
R_{o}=\sqrt{\sqrt{\kappa^{2}+1}+\kappa}
$$

$$
\nu_{e}=2 \sqrt{1-\kappa\left(\sqrt{\kappa^{2}+1}-\kappa\right)}
$$

Because of the nonlinear space charge force the envelope tune $Q_{e}\left(J_{e}\right)$ varies from $Q\left(J_{e}=0\right)=\nu_{e}$ to $Q\left(J_{e} \rightarrow \infty\right) \rightarrow 2$. When $\kappa=0$, the envelope dynamics is linear and $Q_{e}=2$; at the infinite space charge limit $\nu_{e}=\sqrt{2}$.

\section{THE HAMILTONIAN FOR THE PARTICLE PHASE SPACE}

The Hamiltonian for a test particle is given by

$$
\begin{aligned}
H_{p}= & \frac{p_{r}{ }^{2}}{2}+\frac{L^{2}}{2 r^{2}}+\frac{r^{2}}{2}-\frac{\kappa\left(r^{2}-R^{2}\right)}{R^{2}} \Theta(R-r) \\
& -2 \kappa \ln \left(\frac{r}{R}\right) \Theta(r-R)
\end{aligned}
$$

For a beam with mismatch parameter $M \ll 1$ envelope radius changes harmonically with time $R(t)=R_{o}\left(1-M \cos \left(\nu_{e} t\right)\right)$; the particle Hamiltonian can be separated into autonomous Hamiltonian $H_{p o}\left(p_{r}, r\right)$ (can be rewritten in terms of canonical action-angle variables) and time-dependent perturbation $\Delta H\left(p_{r}, r, t\right)$.

$$
\begin{aligned}
H_{p o}\left(p_{r}, r\right)= & \frac{p^{2}}{2}+\frac{L^{2}}{2 r^{2}}+\frac{r^{2}}{2}-\frac{\kappa\left(r^{2}-R_{o}{ }^{2}\right)}{R_{o}{ }^{2}} \Theta\left(R_{o}-r\right) \\
& -2 \kappa \ln \left(\frac{r}{R_{o}}\right) \Theta\left(r-R_{o}\right)
\end{aligned}
$$

$$
\Delta H\left(p_{r}, r, t\right)=-\frac{\kappa\left(r^{2}-R_{o}{ }^{2}\right)}{R_{o}{ }^{2}} M \cos \left(\nu_{e} t\right) \Theta\left(R_{o}-r\right)
$$

Below we consider only $L=0$ case (we replace coordinate $r$ by $y$ ). For a beam with a matched envelope radius $R_{o}$ particle motion inside the core $\left(|y|<R_{o}\right.$ or equivalently $\left.J_{p}<\frac{1}{2}\right)$ is linear with

$$
\nu_{p}=\sqrt{\kappa^{2}+1}-\kappa
$$




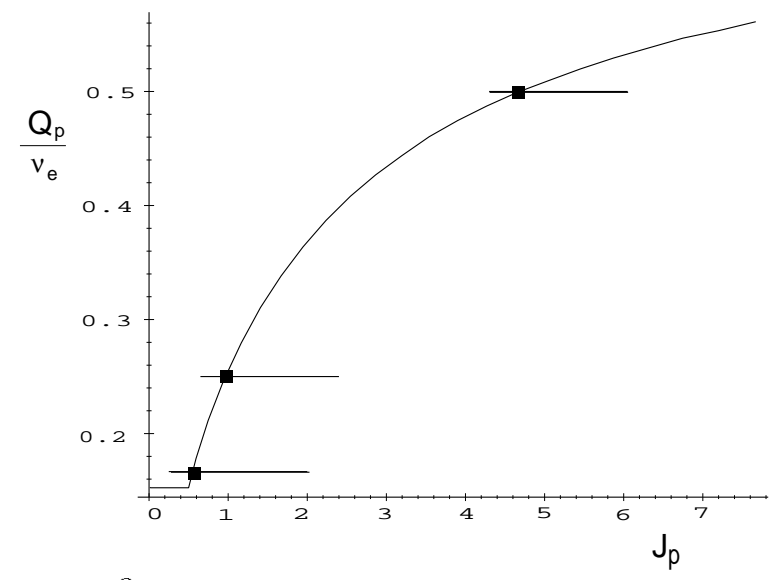

Figure 1. $\frac{Q_{p}}{\nu_{c}}$ vs $J_{p}$ for $\kappa=2.19$. Intersections of horizontal lines with $\frac{Q_{p}}{\nu_{e}}$ mark locations of parametric resonances.

On the other hand in the limit of large amplitude oscillations the space-charge force is not important; particle motion is almost harmonic with $Q_{p}=1$. Thus the tune of a test particle outside the core lies within the range $\left[\nu_{p}, 1\right)$.

Note that functional dependence of particle tune vs. action has a cusp at $r=R_{o}$ (see Figure 1); this suggests that a simple power expansion may not be applicable. On the other hand the sharp rise of the particle tune outside the core will bear important implications to parametric resonances when the system is subject to harmonic perturbations (such as wake fields or envelope modulation).

In terms of action-angle variables the particle Hamiltonian becomes

$$
H_{p}=H_{p o}(J)-M \kappa \cos \left(\nu_{e} t\right) \sum_{n=-\infty}^{\infty} G_{n}(J) e^{i n \psi}
$$

where the strengths of primary resonances $G_{n}(J)$ are

$$
G_{n}(J)=\frac{1}{2 \pi} \int_{-\pi}^{\pi}\left(\frac{y^{2}}{R_{o}{ }^{2}}-1\right) \Theta\left(R_{o}-y\right) e^{-i n \psi} d \psi
$$

Because the Hamiltonian is symmetric with respect to $y \rightarrow-y$ all odd harmonics vanish. The location of primary parametric resonances for particle motion can be found from:

$$
n Q_{p}\left(J_{F P}\right)-\nu_{e}+n N \mathbb{G}_{n}^{\prime}\left(J_{F P}\right)=0
$$

If $M \kappa G_{n}^{\prime}\left(J_{F P}\right)$ is small, the threshold of existence of $n$-th order primary parametric resonance is given by $\frac{\nu_{p}}{\nu_{e}}=\frac{1}{n}$. Therefore as $\kappa$ increases more high order resonances appear (see Figure 2). In general $n$-th order primary resonance exists only if

$$
\kappa>\frac{n^{2}-4}{\sqrt{8\left(n^{2}-2\right)}}
$$

This has an important implication for Halo formation which is enhanced when many high order resonances overlap creating large chaotic area in the phase space. Also as it was found in numerical simulations the resonance strength will increase as well. Finally we see that for a beam with a very large space charge parameter many resonances appear near the core and create a large

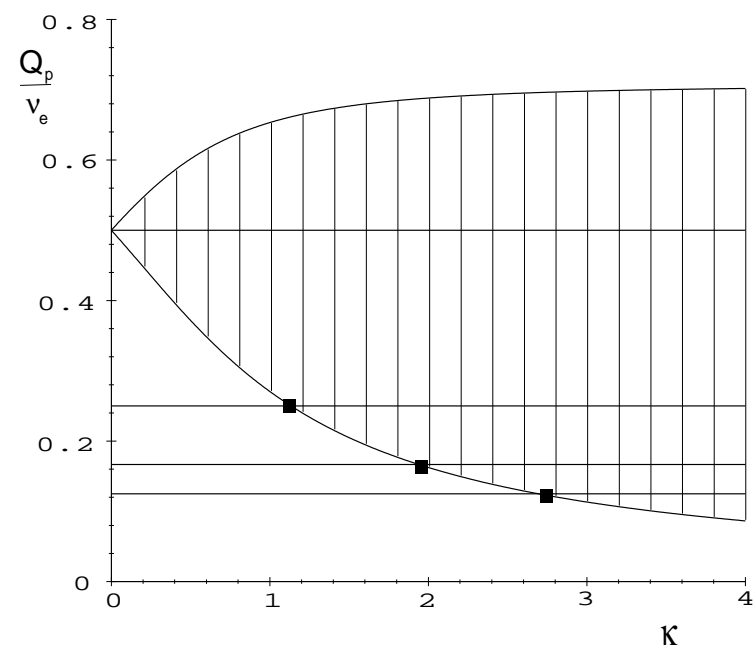

Figure 2. The range of ratios of the particle tune to the envelope tune as function of effective space charge strength $\kappa$.

region of stochasticity. Though motion inside the core is stable, the dynamics of the particle from the diffusive tail is determined by the presence of this chaotic regions.

\section{A. Halo and global chaos}

We examine particle motion in Poincaré surfaces of section at the minimum envelope radius locations; Poincaré energy for particle motion is defined as energy calculated at the minimum radius locations. The Poincaré energy of a test particle outside the core is always larger than $E_{P}=\frac{\nu_{p}}{2(1-M)^{2}}$ maximum energy of core particles. We now consider the motion of a particle with Poincaré energy $E=\eta E_{P}$. For a given mismatch parameter $M$ and $\kappa$ there is a critical number $\eta_{\text {cr }}$ such that all test particles with $\eta>\eta_{c r}$ will orbit about the $2: 1$ resonance (which exists for all values of $\kappa$ ) and thus become halo particles. Numerical simulations indicate that $\eta_{c r}$ is a smooth function of mismatch parameter $M$ (Figure 3 a) if $\kappa<2.2$. However for $\kappa>2.2$, we can always observe very sharp transition when $M$ exceeds some critical value $M_{c r}(\kappa)$ (Figure $3 \mathrm{~b}$ ). When $\kappa$ is small there are few resonances near the core. Since the width of period 2 resonance and width of stochastic layer varies smoothly with the modulation amplitude, $\eta_{c r}$ will decrease smoothly as $M$ increases. On the other hand when $\kappa$ is large there are many primary and secondary resonances and local chaos is formed near the core. Once the stochastic layer layer of period 2 resonance overlaps with local chaos, global chaos occurs.

\section{B. Period 2 resonance and Halo diameter}

It is evident from numerical simulations that Halo diameter is determined mainly by period 2 resonance. Though the location of SFP of this resonance is shifted when modulation amplitude increases, this shift is linearly proportional to $M$ (Figure 4) and is omitted for the sake of simplicity. So we can estimate the maximum transverse energy of Halo particles by $E\left(J_{r}\right)+\frac{\Delta E}{2}$ where $E\left(J_{r}\right)$ is the energy of resonance particle and $\Delta E$ is the energy width of the resonance :

$$
\Delta E=Q_{p}\left(J_{r}\right) \Delta J=4 Q_{p}\left(J_{r}\right) \sqrt{\frac{M G_{2}\left(J_{r}\right)}{Q_{p}^{\prime}\left(J_{r}\right)}}
$$




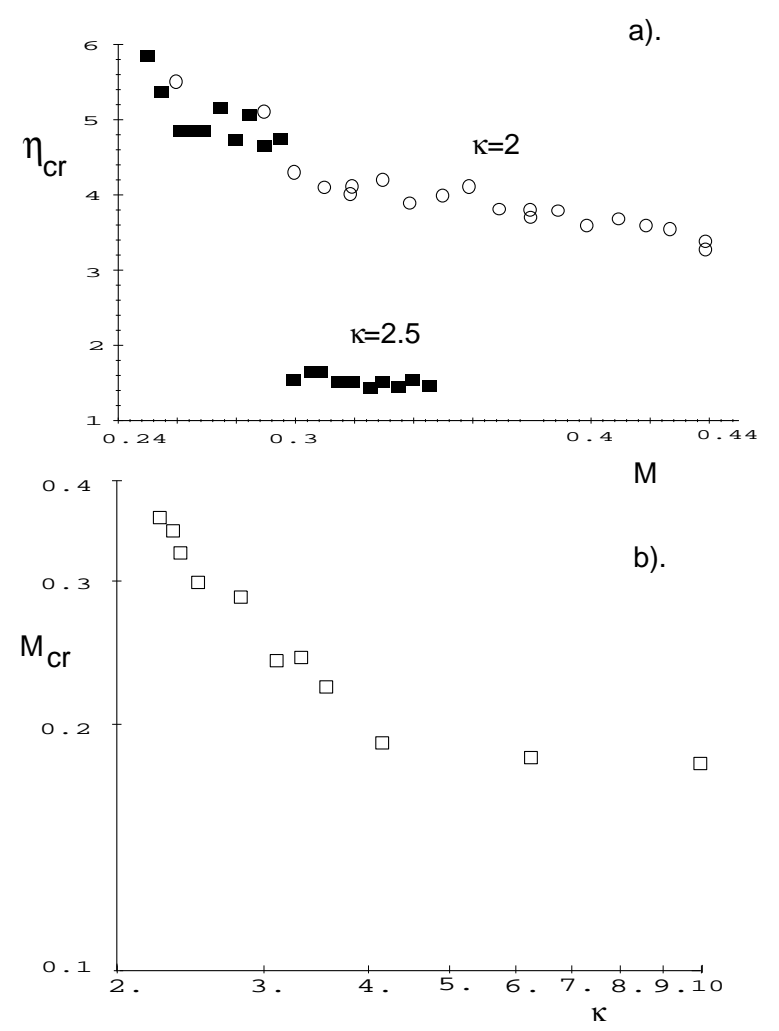

Figure 3. a). $\eta_{c r}$ as function of $M$ for $\kappa=2$ (boxes) and 2.5 (circles). b). $M_{c r}$ vs space charge parameter $\kappa$ obtained from numerical simulations .

Taking advantage of the fact that dynamics of the system is defined by a single parameter $\kappa$ we can use the following approximate formula for maximum transverse energy of Halo particles (Figure 4) :

$$
E_{\text {max }}=\epsilon \mu E_{r}(\kappa)+\epsilon \mu \nu_{e}(\kappa) \sqrt{M} \beta \kappa
$$

where $\beta \approx 2.5$ obtained from numerical simulations.

\section{CONCLUSION}

In conclusion, we studied the beam transport problem solving simultaneously the dynamic equations for the $\mathrm{KV}$ envelope and a test particle. Parametric resonances for particle motion can be generated by a mismatched envelope oscillations. The resonance condition is found to depend only on a single effective space charge parameter $\kappa$. Due to the mismatched beam envelope oscillations, the period 2 resonance occurs at all values of space charge perveance. From our numerical simulations, it is observed that the critical Poincare energy for the halo particle exhibits a sharp transition when plotted as a function of envelope mismatch parameter what is related to the onset of global chaos. The relation between the critical mismatch parameter $M_{c}$ and the effective space charge parameter $\kappa$ is obtained from numerical simulations. Halo diameter is estimated. Effects of angular momentum are considered in other publications [6].

We have studied the halo formation for a space charge dominated beams in a uniform focusing channel. This method can readily be applied to study the beam transport problems in a periodic focusing channel, where Floquet transformations is needed before analytic solution can be obtained [6].

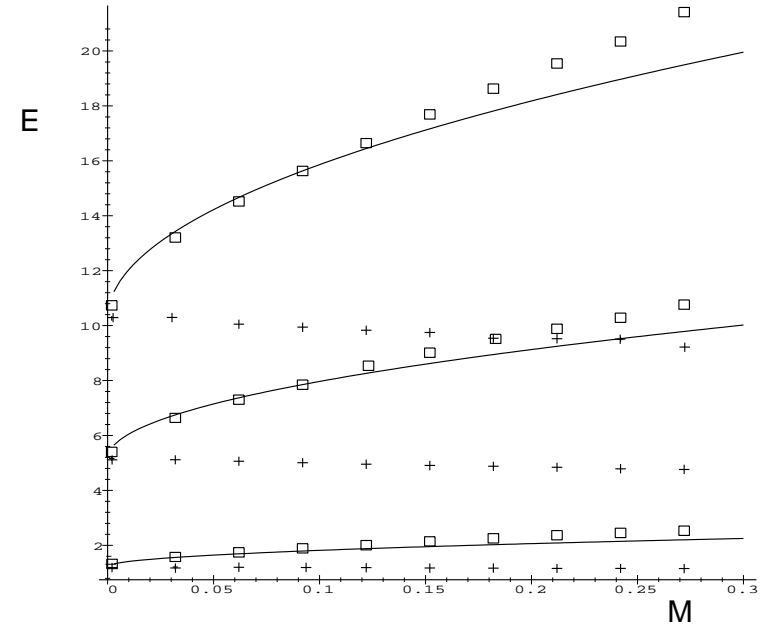

Figure 4. Maximum energy of Halo particles (boxes), equation 14(line) and location of SFP of period 2 resonance (crosses) as function of modulation amplitute $M$ for $\kappa=1.25,6.25,12.5$.

\section{References}

[1] I.M. Kapchinskij and V.V. Vladimirskij, Proceedings of the International Conf. on High Energy Accelerators, p. 274 (CERN, Geneva, 1959).

[2] R.A. Jameson, in Proc. of the 1993 Part. Accel. Conf. p.3926 (IEEE, Piscataway, 1993); Los Alamos Report No. LA-UR93-1209 (1993) (unpublished).

[3] J.S. O' Connell, T.P. Wangler, R.S. Mills and K.R. Crandall, in Proceedings of the Particle Accelerator Conf. edited by J. Bisognano, p.3657 (IEEE, Piscataway, NJ, 1993).

[4] J.M. Lagniel, Nucl. Inst. and Meth. in Phys. Res. A345, 46 (1994), ibid. 405 (1994).

[5] I. Hofmann, L.J. Laslett, L. Smith, and I. Haber, Particle Accelerators, 13, 145 (1983); J. Struckmeier and M. Reiser, Particle Accelerators, 14, 227 (1983).

[6] S.Y.Lee, A.Riabko, Phys.Rev. E51, 1609 (1995); A.Riabko, et al., Phys. Rev. E51, May (1995). 